\title{
Evaluation of five cell-free DNA isolation kits for plasma
}

Zhongzhen Liu ${ }^{1,2}$, Xi Yang ${ }^{1,2}$, Haixiao Chen ${ }^{1,2}$, Sujun $\mathrm{Zhu}^{3}$, Juan Zeng ${ }^{3}$, Fang Chen ${ }^{1,2}$, Wen-Jing Wang ${ }^{1,2 \#}$

1 BGI-Shenzhen, Shenzhen 518083, China.

2 China National GeneBank, BGI-Shenzhen, Shenzhen, China.

3 Obstetrics Department, Shenzhen Maternity and Child Healthcare Hospital, Shenzhen, Guangdong Province, China

\#Corresponding author: wangwenjing@ genomics.cn 


\begin{abstract}
Cell-free DNA (cfDNA) has been widely used in prenatal test and cancer diagnosis nowadays. The cost- and time- effective isolation kits are needed especially in large-scale clinical application. Here, we compared three domestic kits: VAHTS Serum/Plasma Circulating DNA kit (VZ), MagPure Gel Pure DNA mini kit (MG) and Serum/Plasma Circulating DNA Kit (TG), together with QIAamp Circulating Nucleic Acid Kit (QC) and QIAamp DNA Blood Mini Kit (QD) in cfDNA isolation. cfDNA was isolated from the pooled samples with spike-in fragments, qPCR was conducted to quantify the spike-in fragments recovery. The results indicated that all of the five kits could isolate cfDNA with different efficiency. The VZ kit had an efficiency as high as 90 percent, which is comparable to QC kit. The libraries were constructed using the isolated cfDNAs, quantified by Qubit and analyzed by 2100 bioanalyzer. Both showed the libraries were qualified. Finally, cffDNAs were detected by qPCR targeting SRY gene using libraries from pregnant women bearing male fetuses. All five kits could isolate cffDNAs that could be detected by qPCR. Our results provided more choices in wide-scale clinical application of cfDNA-based non-invasive genetic tests.
\end{abstract}

\title{
Key words
}

cell-free DNA; isolation kits; recovery efficiency; liquid biopsy 


\section{Introduction}

Since the discovery of the circulating tumor DNA from cancer patients serum and plasma in 1996 and fetal DNA from maternal serum and plasma in 1997, the circulating cell-free DNA (cfDNA) has gotten widely attention [1-3]. The cfDNAs originate from the apoptosis, necrosis or active release of tumor cells in cancer patients, fetal trophoblastic cells in pregnant women, or donor cells after transplantation [4]. Due to the safety and convenience, cfDNAs have been widely used as biomarkers in non-invasive fetal screening (NIPS), organ transplant graft rejection [5], trauma [6], sepsis [7], myocardial infraction [8].

In general, there are two common ways through which the analysis of cfDNAs are conducted. One way is quantified PCR, including qPCR or digital PCR, which can analyze the target genes with site mutations or even structural variant. The limitation is the mutations or SVs should have been proven to potentially involved in specific cancer or birth defects $[3,9]$. The second way is next generation sequencing, which is competent to detect all of the potential mutations or SVs of the cell-free tumor DNA (ctDNA) or cell-free fetal DNA (cffDNA) from very early stagy, and give valuable information for diagnosis and treatment $[10,11]$.

One of the most important steps of cfDNA analysis is extraction of cfDNAs from the liquid biopsies. It is known that the concentration of cfDNA in plasma is usually very low, ranging from several to tens of nanogram per milliliter [12]. The variety of the concentration is large according to the progression of disease or pregnant terms. Moreover, the 'valuable cfDNA', which means the ctDNA from the tumors or cffDNA from the fetuses only accounts for small proportion of the total cfDNA, especially in early stages at which the diagnosis is more valuable. Given the samples are limited, the efficient cfDNA extraction kits are needed to be developed. Nowadays the most popular kits are QIAamp Circulating Nucleid Acid kits, which can give a stable and high recovery, while the higher cost of the kits limits the massive application. The traditional Triton/Heat/Phenol (THP) method is cost-effective, but costs much time, and is difficult to conducted automatically. 
A series of reports compared the homemade or commercially available cfDNA extraction kits [13-18], which gave valuable guidelines to select the suitable kits for different samples. Still there are limitations in these studies. Most of the kits are less suitable in industrial application due to the time- and money-consumption. In the present study we directly compared three domestic cfDNA extraction kits with QIAamp Circulating Nucleid Acid kits using real-time PCR and Agilent 2100 bioanalyzer. We found that recovery efficiency of cfDNAs by at least one kit was comparable to QIAamp while more cost-effective. Our results gave an additional choice in cfDNA isolation. 


\section{Materials and methods}

\section{Plasma sample collection and pretreatment}

Five $\mathrm{mL}$ peripheral blood were taken using EDTA anticoagulant-coated tubes from pregnant women in the second trimester. All blood samples were centrifuged at low speed (3000 rpm) for $5 \mathrm{~min}$ at $4 \square$ within three hours after collection. The supernatant was centrifuged at high speed $(1,4000 \mathrm{rpm})$ for $15 \mathrm{~min}$ at $4 \square$. The genders of the fetuses were indicated by ultrasound and confirmed by PCR using plasma. The blood samples were fell into three groups: for group I, $1 \mathrm{~mL}$ plasma from six pregnant women bearing female fetuses were pooled together and divided into 15 aliquots and processed by five kits. For group II, $1 \mathrm{~mL}$ plasma from the identical six pregnant women were pooled together and 15,000,000 copies of spike-in-162, 340, 500 and SRY were added, and then divided into 15 aliquots and processed by five kits. For group III, $2.5 \mathrm{~mL}$ plasma from three pregnant women bearing male fetus was processed by these five kits separately. Written consent forms were obtained from all women, and the study was approved by the BGI Institutional Review Board (BGI-IRB17166).

\section{Spike-in fragments preparation}

The spike-in fragments were constructed by insertion the SPUD double strand DNA (5'-AACTTGGCTTTAATGGACCTCCAATTTTGAGTGTGCACAAGCTATGGAA CACCACGTAAGACATAAAACGGCCACATATGGTGCCATGTAAGGATGAATG T-3') into pCE2-TA/Blunt-Zero vector using 5 min TA/Blunt-Zero Cloning Kit (vazyme, C601). The amplicons with different lengths (162, 340, 500bp) from pCE2-TA/Blunt-Zero vector were amplified using the primer pairs: spike-in-162 Fw \& Re, spike-in-340 Fw \& Re or spike-in-500 Fw \& Re. SRY segments was amplified with primer pairs spike-in-SRY Fw \& Re using male genomic DNA as template. After GEL-purification and quantification, 15,000,000 copies of each fragment were added in aliquots in group II. All the primers were listed in table 1.

\section{DNA extraction}

The cfDNA was extracted from $200 \mathrm{uL}$ plasma using VAHTS Serum/Plasma Circulating DNA kit (Vazyme, Cat. No.: N902-01-BOX2, VZ for short), MagPure 
Gel Pure DNA mini kit (Magen, Cat. No.: MD5001-02, MG for short), Serum/Plasma Circulating DNA Kit (TIANGEN, Cat. No.: DP339, TG for short), or from $1 \mathrm{~mL}$ plasma using QIAamp Circulating Nucleic Acid Kit (QIAGEN, Cat. No.: 55114, QC for short) following the manufacturer's guide. We also used QIAamp DNA Blood Mini Kit (QIAGEN, Cat. No.: 51104, QD for short), which was not designed to extract cfDNA. The cfDNA was eluted by $200 \mathrm{uL} \mathrm{TE}$ buffer for QC and $40 \mathrm{uL}$ for the rest.

\section{Quantitative PCR}

qPCR was conducted on StepOnePlus ${ }^{\mathrm{TM}}$ Real-Time PCR System (Applied Biosystem, 4376600) using SYBR Premix Ex Taq (Tli RNaseH Plus), ROX plus (Takara, Cat. No.: RR420LR). The primers for qPCR were listed in table 1.

\section{cfDNA library construction}

The extracted cfDNAs were processed to library using MGIEasy Cell-free DNA Library Prep kit (MGI, cat. No.: AA00226). The Ad153 Fw \& Re adapters listed in table 1 were ligated to the amplicons.

\section{Qubit DNA quantification}

The library was qualified using Qubit dsDNA HS Assay Kit (Invitrogen, cat. No.: Q32851). 2 uL of each library was loaded and the concentration was detected by Qubit 3.0 Fluorometer (Invitrogen).

\section{Bioanalyzer assay}

The libraries were loaded on DNA 1000 series II chip to undergo automated electrophoresis using Agilent 2100 Bioanalyzer (Agilent) for sizing and qualification of the library. 


\section{Results}

\section{The cfDNA yield was comparable between $\mathrm{VZ}$ and QC, with the efficiency more} than $90 \%$

To compare the cfDNA yield directly, we pooled six samples together and divided into 15 tubes, $200 \mathrm{uL}$ each for the $\mathrm{VZ}, \mathrm{MG}, \mathrm{TG}$, QD and $1 \mathrm{~mL}$ each for QC, as the lowest volume as the kit requires. We extracted cfDNA with five kits, each kit had three repeats. Due to the addition of carrier nucleic acids, which could increase the recovery according to the product manual of TG, QC and QD, the concentration of cfDNA in elution could not be detected directly. To overpass this problem, we evaluated the cfDNA yield by using exogenous spike-in fragments. We added a mixture of spike-in-162, 340, 500, spike-in-SRY (162 bp in length), 1,500,000 copies for each segment, to $200 \mathrm{uL}$ plasma or 7,500,000 to $1 \mathrm{~mL}$ plasma for QC. We detected the number of copies of each segment in elution buffer using qPCR, and calculated the recovery efficiency (RE) by using the equation RE = copy number eluted / 1,500,000 (7,500,000 for QC). As shown in figure 1, generally, the RE of QC was highest for all four segments $(100.64 \%, 111.45 \%, 92.41 \%, 68.81 \%)$. The VZ kit displayed similar yield $(96.54 \%, 95.03 \%, 87.93 \%, 73.49 \%)$. The RE of MG kit was lower, while the TG kit was the lowest. The QD kit, which is not designed to isolate cfDNA, also generated weak signals.

There was a relationship between RE and size of segments. The yields of spike-in-162 and spike-in-SRY, both of which are 162bp, was the highest for all of the four kits. On the other hand, spike-in-500 had the lowest RE. It has been proven that $162 \mathrm{bp}$ fragments account for the majority of circulating DNA, thus for QC and VZ kits, nearly $90 \%$ of cfDNA could be isolated.

\section{The isolated cfDNA was qualified for library construction}

Next, we evaluated whether the cfDNAs isolated through these five kits could be used in library construction for NIPT. We extracted cfDNAs from the plasma in the group I using different kits and kept $20 \mathrm{uL}$ elution to conduct library conduction. We eluted the libraries in $20 \mathrm{uL}$ elution buffer, and evaluated the concentration of the libraries. 
As shown in figure 2, cfDNAs from all five kits were qualified to construct library (figure 2). As in spike-in experiments, the concentration of libraries from VZ and QC has the highest concentration, nearly $30 \mathrm{ng} / \mathrm{uL}$. MG and TG kits can reach to more than $10 \mathrm{ng} / \mathrm{uL}$.

We also analyzed the molecular weight and concentration of libraries using 2100 bioanalyzer. As expected, there was a main peak at around 250 and a minor peak at around 430 (figure 3). The tendency was similar with spike-in experiment.

\section{All of the three kits were efficient to detect cell free fetal DNA}

Due to scarcity of cffDNA in mother's plasma, the efficient recovery of cffDNA calls for high efficiency of the kits and no bias between cffDNA and mother's cfDNA. We evaluated the cffDNA isolation efficiency by detecting copy number of $\mathrm{Y}$ chromosomal segments isolated from the plasma of mothers bearing male fetuses. We isolated cfDNA from plasma in group III using these five kits separately and used 20 $\mathrm{uL}$ elution to construct libraries. Then we conducted qPCR using the primer pair identifying SRY fragments and the libraries as templates. Our results indicated that the cfDNAs from all five kits could give signals of SRY fragments (figure 4). We can also calculate the copy numbers of SRY segments in the libraries. We found for all three plasma, the SRY copy numbers from QC and VZ libraries were more than 5000 copies. The copy numbers from MG and TG were smaller, but still enough to detect the fetal genome. 


\section{Discussion}

In this study, we evaluated five isolation kits of cfDNA extraction from plasma of pregnant women. We compared the direct recovery efficiency of these five kits by investigation of exogenous spike-in fragments using qPCR and found all five kits could extract cfDNAs with different efficiency, while the recovery of QC and VZ could reach up to $90 \%$. We also evaluated the libraries constructed from the cfDNAs extracted by these five kits, and all were qualified for library construction.

cfDNA analysis has been proven a powerful tool in cancer diagnosis and NIPS. As the initial step of cfDNA analysis, cfDNA extraction is the most important, because the purification and recovery efficiency determines the following analysis steps and finally the signal quantity. Nowadays there were a series of studies investigating the efficiency of several kits, including QIAamp circulating nucleic acid kit (QIAgen, Valencia, CA, USA), MagNA Pure Compact (MPC) Nucleic Acid Isolation Kit I (Roche Diagnostics, Penzberg, Germany) etc., which are popular worldwide. As the large-scale clinical application of cfDNA analysis developed recently, the lower-cost as well as efficiency-comparable kits are in need, especially in the less developed countries, like China. Thus, in this study, we focused on three domestic DNA extraction kits, VZ, MG and TG, which are less expensive.

Another factor that influences large-scale application is the automation. At present there are two technologies employed in commercial cfDNA purification kits: spin column-based and magnetic beads-based approach. Compared to column, the magnetic beads-based approach could be conducted in 96-well plates automatically and has been applied in large-scale cfDNA extraction. Indeed, the VZ and MG kits has already been used in automatic extraction platform and performed stably.

Notably, the recovery efficiency of spike-in calculated by qPCR was higher than $100 \%$, probably due to the systematic error of qPCR, which is not easily avoided. The more exact method was digital PCR, which we are trying to establish.

It has been proven that there are a dominant peak at approximate $162 \mathrm{bp}$ and a minor peak at around 340 bp [19]. We did not run 2100 after cfDNA extraction directly due 
to addition of carrier nucleic acids according to the manual of TG, QC and QD. Alternatively, we run 2100 after library construction, when the 90bp adapters were added to the cfDNAs. There should be a major peak at $\sim 250 \mathrm{bp}$ and a minor peak at 430bp. Our 2100 bioanalyzer results were in accordance with this conclusion. It is reasonable to use $162 \mathrm{bp}-, 340 \mathrm{bp}-, 500 \mathrm{bp}$ - spike-in fragments, which represent the main parts of the cfDNAs, in our experiments. For QC and VZ, the recovery efficiency of $162 \mathrm{bp}$ and $340 \mathrm{bp}$ fragments was more than $90 \%$, so we can give a bold prediction that these two kits have a RE of at least 90 percent.

As NIPS is widely used in prenatal screening, the cost- and time- efficient cfDNA extraction methods are valuable in widescale application. Our results may provide more choices in a clinical setting.

\section{Acknowledgement}

We are grateful to the participants who donated their samples to our project. This project is supported by the National Natural Science Foundation of China (No.81300075), the Natural Science Foundation of Guangdong Province (No.

2014A030313795), the Shenzhen Municipal Government of China (No.JCYJ20170412152854656, JCYJ20180703093402288). 


\section{References}

1. Nawroz H, Koch W, Anker P, Stroun M, Sidransky D. Microsatellite alterations in serum DNA of head and neck cancer patients. Nat Med. 1996;2(9):1035-7. PubMed PMID: 8782464.

2. Chen XQ, Stroun M, Magnenat JL, Nicod LP, Kurt AM, Lyautey J, et al. Microsatellite alterations in plasma DNA of small cell lung cancer patients. Nat Med. 1996;2(9):1033-5. PubMed PMID: 8782463.

3. Lo YM, Corbetta N, Chamberlain PF, Rai V, Sargent IL, Redman CW, et al. Presence of fetal DNA in maternal plasma and serum. Lancet. 1997;350(9076):485-7. doi: 10.1016/S0140-6736(97)02174-0. PubMed PMID: 9274585.

4. Stroun M, Lyautey J, Lederrey C, Olson-Sand A, Anker P. About the possible origin and mechanism of circulating DNA apoptosis and active DNA release. Clin Chim Acta. 2001;313(1-2):139-42. PubMed PMID: 11694251.

5. Gwinner W. Renal transplant rejection markers. World J Urol. 2007;25(5):445-55. doi: 10.1007/s00345-007-0211-6. PubMed PMID: 17786452.

6. Lo YM, Rainer TH, Chan LY, Hjelm NM, Cocks RA. Plasma DNA as a prognostic marker in trauma patients. Clin Chem. 2000;46(3):319-23. PubMed PMID: 10702517.

7. Martins GA, Kawamura MT, Carvalho Mda G. Detection of DNA in the plasma of septic patients. Ann N Y Acad Sci. 2000;906:134-40. PubMed PMID: 10818609.

8. Chang CP, Chia RH, Wu TL, Tsao KC, Sun CF, Wu JT. Elevated cell-free serum DNA detected in patients with myocardial infarction. Clin Chim Acta. 2003;327(1-2):95-101. PubMed PMID: 12482623.

9. Vasioukhin V, Anker P, Maurice P, Lyautey J, Lederrey C, Stroun M. Point mutations of the $\mathrm{N}$-ras gene in the blood plasma DNA of patients with myelodysplastic syndrome or acute myelogenous leukaemia. Br J Haematol. 1994;86(4):774-9. PubMed PMID: 7918071.

10. Volckmar AL, Sultmann H, Riediger A, Fioretos T, Schirmacher P, Endris V, et al. A field guide for cancer diagnostics using cell-free DNA: From principles to practice and clinical applications. Genes Chromosomes Cancer. 2018;57(3):123-39. doi: 10.1002/gcc.22517. PubMed PMID: 29205637.

11. Bianchi DW, Chiu RWK. Sequencing of Circulating Cell-free DNA during Pregnancy. N Engl J Med. 2018;379(5):464-73. doi: 10.1056/NEJMra1705345. PubMed PMID: 30067923.

12. Fleischhacker M, Schmidt B. Circulating nucleic acids (CNAs) and cancer--a survey. Biochim Biophys Acta. 2007;1775(1):181-232. doi: 10.1016/j.bbcan.2006.10.001. PubMed PMID: 17137717.

13. Sorber L, Zwaenepoel K, Deschoolmeester V, Roeyen G, Lardon F, Rolfo C, et al. A Comparison of Cell-Free DNA Isolation Kits: Isolation and Quantification of Cell-Free DNA in Plasma. J Mol Diagn. 2017;19(1):162-8. doi: 10.1016/j.jmoldx.2016.09.009. PubMed PMID: 27865784.

14. Perez-Barrios C, Nieto-Alcolado I, Torrente M, Jimenez-Sanchez C, Calvo V, Gutierrez-Sanz $\mathrm{L}$, et al. Comparison of methods for circulating cell-free DNA isolation using blood from cancer patients: impact on biomarker testing. Transl Lung Cancer Res. 2016;5(6):665-72. doi: 10.21037/tlcr.2016.12.03. PubMed PMID: 28149760; PubMed Central PMCID: PMCPMC5233878.

15. Jain M, Balatsky AV, Revina DB, Samokhodskaya LM. Direct comparison of QIAamp DSP 
Virus Kit and QIAamp Circulating Nucleic Acid Kit regarding cell-free fetal DNA isolation from maternal peripheral blood. Mol Cell Probes. 2019;43:13-9. doi: 10.1016/j.mcp.2018.12.006. PubMed PMID: 30584912.

16. Fleischhacker M, Schmidt B, Weickmann S, Fersching DM, Leszinski GS, Siegele B, et al. Methods for isolation of cell-free plasma DNA strongly affect DNA yield. Clin Chim Acta. 2011;412(23-24):2085-8. doi: 10.1016/j.cca.2011.07.011. PubMed PMID: 21861994.

17. Xue X, Teare MD, Holen I, Zhu YM, Woll PJ. Optimizing the yield and utility of circulating cell-free DNA from plasma and serum. Clin Chim Acta. 2009;404(2):100-4. doi: 10.1016/j.cca.2009.02.018. PubMed PMID: 19281804.

18. Devonshire AS, Whale AS, Gutteridge A, Jones G, Cowen S, Foy CA, et al. Towards standardisation of cell-free DNA measurement in plasma: controls for extraction efficiency, fragment size bias and quantification. Anal Bioanal Chem. 2014;406(26):6499-512. doi: 10.1007/s00216-014-7835-3. PubMed PMID: 24853859; PubMed Central PMCID: PMCPMC4182654.

19. Fan HC, Blumenfeld YJ, Chitkara U, Hudgins L, Quake SR. Analysis of the size distributions of fetal and maternal cell-free DNA by paired-end sequencing. Clin Chem. 2010;56(8):1279-86. doi: 10.1373/clinchem.2010.144188. PubMed PMID: 20558635. 


\section{Figure legends}

Figure 1. Comparison of five cfDNA isolation kits by using the percentage of spike-in recovery generated by VZ, MG, TG, QC, QD. Four spike-in fragments with indicated lengths were added in the pooled plasma. Three replicates were conducted for each kit. qPCR was conducted to calculate the copy numbers using standard curve method. Note that for some fragments the efficiency was higher than 100 percent, probably due to the systematic errors of qPCR.

Figure 2. The concentration of libraries constructed from cfDNAs isolated by five kits from pooled plasma. Three replicates were conducted for each kit. And $20 \mathrm{uL}$ elution was used to construct libraries.

Figure 3. The 2100 bioanalyzer results of libraries constructed from cfDNAs isolated by five kits from pooled plasma. One library of each kit was loaded.

Figure 4. The copy numbers of SRY in $1 \mathrm{uL}$ libraries constructed from cfDNA isolated by five kits from three pregnant women bearing male fetuses. 
bioRxiv preprint doi: https://doi.org/10.1101/715821; this version posted July 26,2019 . The copyright holder for this preprint (which was not certified by peer review) is the author/funder, who has granted bioRxiv a license to display the preprint in perpetuity. It is made available under aCC-BY 4.0 International license. 


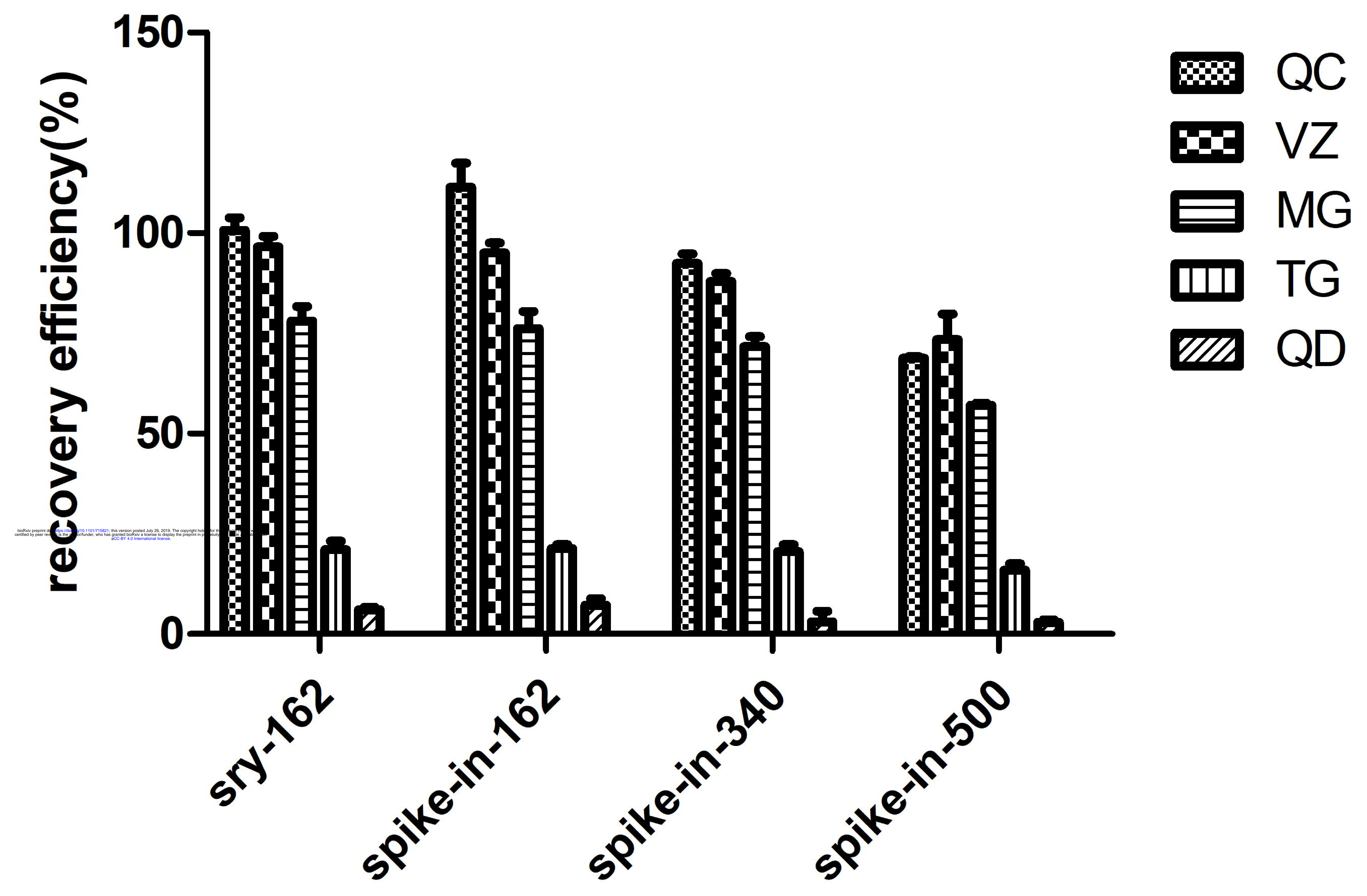




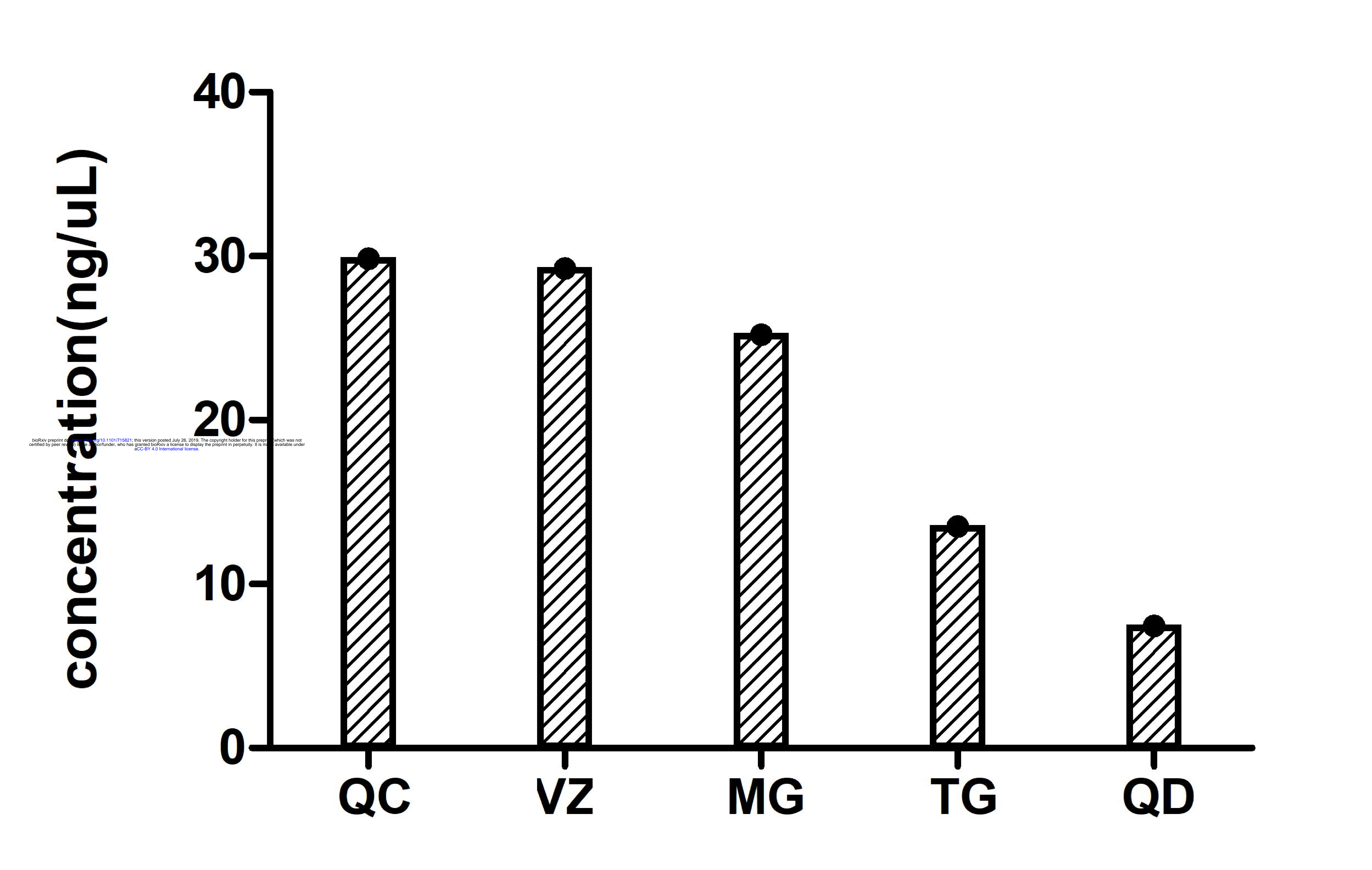




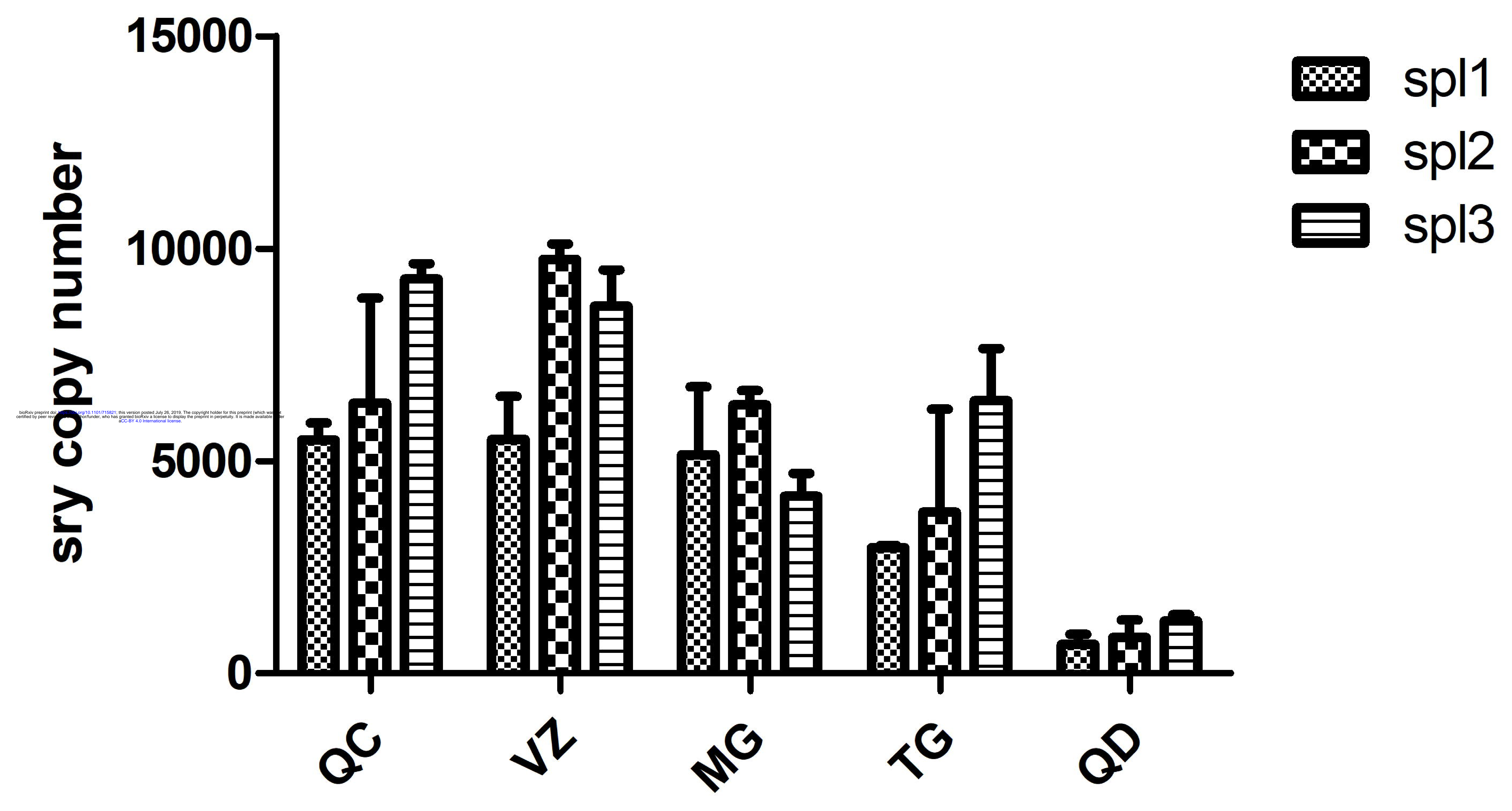


Table 1. primers used in this study

\begin{tabular}{|c|c|}
\hline Primer name & Sequences $\left(5^{\prime} \rightarrow 3^{\prime}\right)$ \\
\hline Spike-in-162 fw & GTCCTGCAGGTTTAAACG \\
\hline Spike-in-162 re & AATTGAATTTAGCGGCCGCG \\
\hline Spike-in-340 fw & TGATCTCAGCCACCGCGAAA \\
\hline Spike-in-340-re & AGCCATCCAGTTTACTTTG \\
\hline Spike-in-500-fw & GGATGGCTTTCTCGCCGC \\
\hline Spike-in-500-re & GCGATGTTTCGCTTGGTGG \\
\hline SRY-fw & CCCGAATTATAAGTATCGAC \\
\hline SRY-re & TGCTCCATTCTTGAGTGTG \\
\hline Spike-in-162 qPCR fw & AACTTGGCTTTAATGGACCTCCA \\
\hline Spike-in-162 qPCR re & ACATTCATCCTTACATGGCACCA \\
\hline Spike-in-340 qPCR fw & TAGAAAGCCAGTCCGCAGAA \\
\hline Spike-in-340 qPCR re & TCCATAAAACCGCCCAGTCT \\
\hline Spike-in-500 qPCR fw & GTGCCCTGAATGAACTGCAA \\
\hline Spike-in-500 qPCR re & ATAGCAGCCAGTCTCTTCCC \\
\hline SRY qPCR fw & AGTATCGACCTCGTCGGAAG \\
\hline SRY qPCR re & TCTTGAGTGTGTGGCTTTCG \\
\hline Library adapter AD153 fw & TTGTCTTCCTAAGGAACGACATGGCTACGATCCGACTT \\
\hline $\begin{array}{l}\text { Library adapter AD153 re } \\
\text { with barcode }\end{array}$ & $\begin{array}{l}\text { AGTCGGAGGCCAAGCGGTCTTAGGAAGACAAXXXXXXXXXX } \\
\text { CAACTCCTTGGCTCACA }\end{array}$ \\
\hline
\end{tabular}

